\title{
Extracardiac Imaging Findings in COVID-19 Associated Multisystem Inflammatory Syndrome in Children
}

Edward Patrick Fenlon, M.D. ( $\nabla$ ef2599@cumc.columbia.edu )

Columbia University Medical Center

\section{Susie Chen, MD}

Columbia University Medical Center

Carrie Ruzal-Shapiro, MD

Columbia University Medical Center

Diego Jaramillo, MD, MPH

Columbia University Medical Center

Alexis B.R. Maddocks, MD

Columbia University Medical Center

\section{Research Article}

Keywords: COVID-19, Multisystem Inflammatory Syndrome in Children (MIS-C), Pediatric Multisystem Inflammatory Syndrome temporally associated with COVID-19 (PMIS-TS or PMIS), Kawasaki disease

DOl: https://doi.org/10.21203/rs.3.rs-42892/v1

License: (c) (1) This work is licensed under a Creative Commons Attribution 4.0 International License. Read Full License 


\section{Abstract}

Background - COVID-19 associated Multisystem Inflammatory Syndrome in Children (MIS-C) is an emerging disease that presents with inflammation of multiple organs. The extracardiac radiographic manifestations of this syndrome are not well understood.

Objective - We reviewed the radiologic findings of MIS-C in a cohort of children with a confirmed diagnosis of the syndrome.

Materials and methods - In a retrospective study from 4/1/2020 to 5/22/2020, we reviewed imaging studies of 38 children with MIS-C, 21 females (55\%) and 17 males (45\%), with an average age of 9.2 years (range $1.3-20$ years). Thirty six had chest radiographs, 6 had abdominal radiographs, 12 had abdominal sonograms or MRI, 2 had neck sonograms, and 3 had brain MRI.

Results - 28 patients had pulmonary disease, with $24(86 \%)$ having bilateral opacities, mostly diffuse $(n=16,57 \%)$. The most common abnormalities were peribronchial thickening $(n=19,68 \%)$, ground glass opacities $(n=15,54 \%)$ and consolidation ( $n=5,18 \%)$. Pleural effusions were only present in children 10 years of age and under, and ground glass opacities were seen more often in older patients (59\% vs. $26 \%$, $P<0.05)$. On abdominal imaging, small volume ascites was the most common finding $(n=6,50 \%)$. Other findings included right lower quadrant bowel wall thickening $(n=3,25 \%)$, gallbladder wall thickening $(n=3$, $25 \%)$, and cervical $(n=2)$ or abdominal $(n=2)$ lymphadenophathy. Of 3 patients with brain MRI, one had bilateral parietooccipital abnormalities and another papilledema.

Conclusion - COVID-19 associated MIS-C causes a constellation of findings in the chest and abdomen, most often showing bilateral diffuse pulmonary abnormalities and small volume ascites.

\section{Introduction}

While the coronavirus disease 2019 (COVID-19) pandemic continues to pose a serious threat to adults and particularly the elderly across the globe, children have so far made up a small minority of patients seriously affected by the disease. As of June 6,2020, cumulative hospitalization rates in the United States since March 1, 2020 are only 5.6 per 100,000 patients in children ages 0 to 4 years old and 3.1 per 100,000 in children ages 5 to 17 years old, compared to a rate of 89 per 100,000 in all ages and up to 273 per 100,000 in adults older than 65 [1]. However, the true prevalence of the disease in children is likely higher as the majority of children with active infections are either asymptomatic or only mildly affected [2].

In late April 2020, doctors in the United Kingdom reported an increased incidence of previously healthy children presenting with a severe hyperinflammatory syndrome similar to Kawasaki disease and testing positive for a current or recent COVID-19 infection [3]. This syndrome has since been termed Multisystem Inflammatory Syndrome in Children (MIS-C) by the United States Centers for Disease Control and Prevention (CDC), and as Pediatric Multisystem Inflammatory Syndrome Temporally Associated with 
COVID-19 (PMIS-TS or PMIS) by the Royal College of Paediatrics and Child Health $[4,5]$.

Symptoms typically consist of a combination of fever, abdominal pain, rash,

and conjunctivitis, with laboratory evaluation consistently showing elevated inflammatory markers, and cardiac evaluation often demonstrating hypotensive shock, myocardial dysfunction and occasionally coronary artery dilation or aneurysms. Importantly, few of these children present with respiratory symptoms, distinguishing it from the typical acute infectious presentation of COVID-19 [6].

This syndrome has quickly emerged as a serious delayed manifestation of COVID-19 infection in children, and radiologists should be aware of the associated clinical features and imaging findings to better aid clinicians in making a timely diagnosis of a syndrome whose presentation can mimic other pathologies. Independent studies and consensus statements have evaluated imaging findings in acute COVID-19 infections in children [7,8], and a small cohort study has reported terminal ileitis in the associated hyperinflammatory syndrome [9]. We sought to review the extracardiac imaging findings of COVID-19 associated MIS-C in a cohort of children with a confirmed diagnosis of the syndrome.

\section{Methods And Materials}

This was a retrospective study performed at a quaternary care children's hospital in New York City with Institutional Review Board (IRB) approval that waived requirements for informed consent.

Subjects

Pediatric patients less than 21 years of age who were admitted through the emergency department or as a transfer from an outside hospital and subsequently diagnosed with MIS-C during the period between April 1, 2020 and May 22, 2020 were included. Diagnosis of MIS-C was made by the inpatient clinical services based on clinical presentation and CDC guidelines, which include: fever, laboratory evidence of inflammation, clinically severe illness requiring hospitalization and multisystem organ involvement in the setting of a current or recent COVID-19 infection based on a reverse transcription polymerase chain reaction (RT-PCR), serology or antigen test, or COVID-19 exposure within 4 weeks of onset of symptoms [4]. All included patients were verified in consultation with our institution's pediatric infectious disease and pediatric rheumatology services.

All patients had either COVID-19 RT-PCR testing, serology testing or both. Patients presenting with acute COVID-19 infection symptoms were excluded. A total of 42 patients were diagnosed with MIS-C during the time period. 38 of these 42 patients underwent some form of imaging in the radiology department during their admission and were included in our study population. Patient electronic medical record charts were queried to identify relevant patient information (age, sex, COVID-19 testing results, recent pneumonia or flu-like illness, presenting symptoms on admission, past medical history, intensive care unit (ICU) admission, discharge status, and imaging studies performed during admission excluding echocardiography).

Image Analysis 
A Picture Archiving and Communications System (PACS) was queried to review all imaging studies performed in the patient population during admission. It should be noted that echocardiographic imaging was performed in all patients but not reviewed for this study.

All imaging studies were initially interpreted in a clinical setting during patient admission by a fellowship trained pediatric radiologist. Each exam was then independently re-reviewed by a fellowship trained pediatric radiologist blinded to patient clinical data.

Chest radiograph findings were classified according to Fleischner society terminology [10]. The findings were characterized according to distribution (unilateral or bilateral); focality (focal, multifocal or diffuse); and characteristics (ground glass opacity, consolidation, peribronchial thickening or septal thickening). If patients had multiple chest radiographs during their admission, all exams were reviewed and findings were based on a summary of findings at the height of the patient's illness.

Statistical Analysis

In the 36 children who had chest radiographs, we compared the imaging findings in the children 10 years of age and under (19 total) with patients over 10 years of age (17 total). Differences between the two groups were compared using the Z-test. We used the same method to compare the most common abdominal findings in 12 patients who had undergone abdominal sonogram or MRI. A p value of 0.05 was considered to be significant.

\section{Results}

\section{Patient Population}

A total of 38 patients were diagnosed with MIS-C and had an imaging study during admission (Table 1). Our patient population consisted of 21 females (55\%) and 17 males (45\%), with an average age of 9.2 years (range $1.3-20$ years). 30 out of 38 (87\%) of our patients had no past medical history. Five patients had mild or intermittent asthma, one had a history of developmental delay, one had a history of meningitis and urinary tract infections as an infant, and one had a history of febrile seizures as an infant. All patients except for 2 tested positive for COVID-19 on either or both RT-PCR and serology testing, with 32 of 38 patients (84\%) testing either negative on RT-PCR but positive on serology, or positive on both. Both patients with negative COVID-19 testing had clinical presentations typical for the syndrome and exposure to family members who either tested positive for COVID-19 or had symptoms of COVID-19. One of these patients had an indeterminate serology result which was not retested.

All patients presented with some combination of symptoms known to occur in MIS-C, including fever, abdominal pain, rash, and conjunctivitis. Many of the patients also presented in hypotensive shock with 30 out of 38 patients being admitted to the ICU. Three patients also presented with cervical lymphadenopathy on physical exam with two undergoing imaging evaluation. Notably, only two patients presented with pulmonary symptoms; one patient with chest pain and dyspnea, and another with 
cough. Some more atypical presentations included two patients with cranial nerve VI palsy, one patient with a large pericardial effusion, and one patient with knee swelling, hematemesis and hematochezia in addition to more typical symptoms. All patients were ultimately discharged home.

Imaging Findings

Chest

Of 36 patients who underwent chest radiography, 8 had normal chest radiographs. In the 28 patients with pulmonary disease, involvement was bilateral in $22(79 \%)$ and unilateral in $4(14 \%)$, with 2 patients having both bilateral and unilateral disease. There were diffuse findings in $18(64 \%)$, multifocal in 4 (14\%) and focal in $6(21 \%)$. The most common type of abnormality was peribronchial thickening $(n=19,68 \%)$, followed by ground glass opacities $(n=15,54 \%)$ and consolidation $(n=5,18 \%)$. One patient had septal thickening, 2 had pleural effusion, and 4 had cardiomegaly.

When the children 10 years of age and under $(n=19)$ were compared with those over 10 years of age $(n=$ 17) we found that effusions were only present in the younger group. Bilateral diffuse disease was the most common presentation in both groups. Ground glass opacities were seen in 5 of $19(26 \%)$ of the younger children and in 10 of $17(59 \%)$ of the older patients $(P<0.05)$. There was no statistical difference in the proportion of patients with consolidation or peribronchial thickening between both groups.

\section{Abdomen}

In the 6 patients who underwent abdominal radiographs, 2 patients had findings suggestive of ileus. In the 12 patients who had abdominal cross-sectional imaging, 6 patients had small volume ascites, 3 had gallbladder wall thickening ( $>4 \mathrm{~mm}), 2$ had gallbladder sludge, 3 had right lower quadrant bowel wall thickening and inflammation, and 2 had right lower quadrant lymphadenopathy. Notably, the liver, spleen and kidneys appeared normal in all of these patients.

Of the children ages 10 and under with abdominal imaging, 4 of 7 (57\%) had ascites, and of those over age 10, 2 of $5(40 \%)$ had ascites. This difference was not statistically significant.

Head and Neck

Two patients with suspected lymphadenopathy on physical exam had sonographic evaluation confirming the presence of cervical lymphadenopathy, with one patient showing a conglomeration of necrotic lymph nodes. Three patients underwent brain MRI. One of these patients with fluctuating mental status that eventually improved had FLAIR (fluid-attenuated inversion recovery) hyperintensity/restricted diffusion involving the bilateral parietooccipital cortices with mild cortical thickening, and punctate T2/FLAIR hyperintensity in the left frontoparietal centrum semiovale. A second patient's brain MRI requested for evaluation of vision complaints and cranial nerve VI palsy demonstrated MR findings compatible with papilledema. A third patient's brain MRI requested for blurred vision and cranial nerve VI palsy demonstrated no abnormality. 


\section{Discussion}

In the months following the onset of the COVID-19 pandemic in Europe, reports of an unusual hyperinflammatory illness in children similar to Kawasaki disease began to emerge in the United Kingdom and Italy $[3,11,12]$. In early May 2020, the New York City Department of Health and Mental Hygiene began receiving reports of cases of a Kawasaki-like disease similar to reported cases in Europe, and by May 12, 2020, 102 children with the syndrome had been identified in New York City [4]. Despite its seemingly unrelated constellation of presenting symptoms and variable effects on a multitude of inflammatory markers and organ function, MIS-C is largely a clinical diagnosis due to its temporal occurrence soon after a COVID-19 infection and its consistent presentation amongst patients of different ages. For this reason, recent reports have focused on the clinical presentation, laboratory work-up, and clinical outcome of children with the syndrome without much comment on associated imaging findings. Our experience, however, provides several insights.

Early on in our hospital's experience with children presenting with MIS-C, just as it was becoming a recognized syndrome in several European countries, imaging was important in excluding other acute pathologies that could also present with fever and abdominal pain, particularly appendicitis and/or ovarian torsion. The absence of acute pathology on imaging prompted the admitting clinicians to think outside of the box, and a careful review of COVID-19 exposure, review of systems and physical exam revealed other signs and symptoms that more aligned with a Kawasaki-like disease.

None of the patients in this population were symptomatic for COVID-19 pneumonia or had a preceding flu-like illness, so presumably the chest radiographic findings reflect MIS-C. Importantly, chest radiograph findings differ from those reported recently for COVID-19 pneumonia [7,8], as most patients had bilateral diffuse disease, with peribronchial thickening and ground glass opacity as the most common findings on chest radiographs. At the time of clinical diagnosis, these findings were favored to reflect cardiogenic and non-cardiogenic pulmonary edema.

Intestinal ileus was described in two patients with abdominal radiographs, and bowel thickening and inflammation in the right lower quadrant, gallbladder wall thickening or sludge, and ascites were the only findings described in patients who underwent abdominal sonograms or MRI. Interestingly, none of our sonograms demonstrated abnormalities of the liver, spleen or kidneys although many patients suffered from multi-organ failure evident clinically. Similar findings of right lower quadrant intestinal inflammation were reported in a cohort of patients with COVID-19 from the United Kingdom who presented with symptoms of atypical appendicitis before rapidly deteriorating as part of a hyperinflammatory syndrome [9].

Pleural effusions and ascites were more frequent in children 10 years of age and under, however the comparisons are not statistically significant rendering their importance difficult to assess.

Although the two main reported indications for ordering sonograms were abdominal pain and/or acute kidney injury, ultimately 9 out of 12 sonograms were ordered as part of an initial work-up for a suspected 
hyperinflammatory Kawasaki-like disease. Two patients who presented early in the cohort had abdominal sonograms ordered to exclude appendicitis in the setting of abdominal pain. Both of these abdominal sonograms demonstrated findings that were equivocal for appendicitis, which prompted a surgical consult and abdominal MRI. One sonogram demonstrated a borderline enlarged appendix (7 mm in diameter), inflammatory changes in the right lower quadrant and distal ileal thickening, which were initially interpreted by an overnight resident as equivocal for appendicitis prompting an abdominal MRI. The subsequent MRI was interpreted as showing a normal appendix but also terminal ileal and rectosigmoid mural thickening, enhancement and edema more typical of inflammatory bowel disease [Fig 2]. The other abdominal sonogram showed ascites and right lower quadrant lymphadenopathy, prompting a surgical consult and abdominal MRI, which showed a normal appendix but persistent unexpected ascites.

Head and neck imaging was performed in a minority of our patients. Targeted neck sonography confirmed two cases of cervical lymphadenopathy suspected on physical exam, with one case demonstrating a cluster of necrotic cervical lymph nodes [Fig 4]. One patient with fluctuating mental status that eventually improved, underwent MRI of the brain which demonstrated FLAIR hyperintensity/restricted diffusion and mild cortical thickening involving the bilateral parietooccipital cortices, and punctate T2/FLAIR hyperintensity in the left frontoparietal centrum semiovale. These findings may have represented an atypical presentation of posterior reversible encephalopathy syndrome (PRES) in the setting of COVID-19 related MIS-C with thrombotic microangiopathy or less likely PRES related to immunosuppressive therapy started during admission.

Just as MIS-C shares commonalities with the clinical presentation of Kawasaki disease, there are many similarities between their extracardiac imaging findings. Typical imaging findings that may be seen in Kawasaki include pulmonary airspace opacities, peribronchial thickening and pleural effusions on chest radiography, and cervical lymphadenopathy on neck imaging with lymph node necrosis on biopsy. Less commonly reported imaging findings in the abdomen include gallbladder hydrops, ascites, ileus, bowel wall edema, abdominal lymphadenopathy and intussusception. None of our patients had significant gallbladder hydrops on abdominal imaging, but three did have gallbladder wall thickening, which is not typically described in Kawasaki disease [13-15].

Our study is limited by the fact that imaging was acquired in each patient as clinically indicated, as there is no routine extracardiac imaging protocol for MIS-C. Besides chest radiographs, advanced imaging was rarely obtained in our patient population. When imaging was obtained, findings were variably present. In fact, 4 out of the original 42 patients diagnosed with the syndrome during the time period had no radiologic imaging (excluding echocardiograms), 16 of 36 had only chest radiographs, and 8 of 36 chest radiographs were normal.

\section{Conclusion}


In conclusion, COVID-19 associated MIS-C is primarily suspected on the basis of clinical presentation rather than imaging findings. However, it should be considered by the clinical team when there are bilateral diffuse pulmonary findings, unexplained ascites or evidence of bowel or gallbladder inflammation in a patient with a history of COVID-19 infection or recent COVID-19 exposure.

\section{Declarations}

Funding: Not applicable

Conflicts of Interest/Competing interests: The authors report no conflicts of interest

Ethics approval: Retrospective study performed with Institutional Review Board (IRB) approval which waived requirements for informed consent.

Consent to participate: Not applicable

Consent for publication: Not applicable

Availability of data and material: Not applicable

Code availability: Not applicable

Author's contributions: Not applicable

\section{References}

1. National Center for Immunization and Respiratory Diseases (NCIRD) Division of Viral Diseases (2020) COVIDView: A Weekly Surveillance Summary of U.S. COVID-19 Activity. Centers for Disease Control and Prevention. https://www.cdc.gov/coronavirus/2019-ncov/coviddata/covidview/index.html. Accessed 14 June 2020

2. Mehta NS, Mytton OT, Mullins E, Fowler TA, Falconer CL, Murphy OB, Langenberg $C$, Jayatunga W, Eddy DH, Nguyen-Van-Tam JS (2020) SARS-CoV-2 (COVID-19): What do we know about children? A systematic review. Clinical Infectious Diseases. Advance online publication. https://doi.org/10.1093/cid/ciaa556

3. Riphagen S, Gomez X, Gonzalez-Martinez C, Wilkinson N, \& Theocharis P (2020) Hyperinflammatory shock in children during COVID-19 pandemic. The Lancet 395(10237):1607-1608. https://doi.org/10.1016/S0140-6736(20)31094-1

4. CDC Health Alert Network (2020) Multisystem Inflammatory Syndrome in Children (MIS-C) Associated with Coronavirus Disease 2019 (COVID-19). Centers for Disease Control and Prevention. https://emergency.cdc.gov/han/2020/han00432.asp. Accessed 10 June 2020

5. Royal College of Paediatrics and Child Health - Health Policy Team (2020) Guidance: Paediatric multisystem inflammatory syndrome temporally associated with COVID-19 (PIMS). Royal College of 
Paediatrics and Child Health. https://www.rcpch.ac.uk/resources/guidance-paediatric-multisysteminflammatory-syndrome-temporally-associated-covid-19. Accessed 10 June 2020

6. Whittaker E, Bamford A, Kenny J, Kaforou M, Jones CE, Shah P, Ramnarayan P, Fraisse A, Miller O, Davies P, Kucera F, Brierley J, McDougall M, Carter M, Tremoulet A, Shimizu C, Herberg J, Burns JC, Lyall H, Levin M, PIMS-TS Study Group and EUCLIDS and PERFORM Consortia (2020) Clinical Characteristics of 58 Children With a Pediatric Inflammatory Multisystem Syndrome Temporally Associated With SARS-CoV-2. JAMA. Advance online publication.

https://doi.org/10.1001/jama.2020.10369

7. Foust AM, Phillips GS, Chu WC, Daltro P, Das KM, Garcia-Peña P, Kilborn T, Winant AJ, Lee EY (2020) International Expert Consensus Statement on Chest Imaging in Pediatric COVID-19 Patient Management: Imaging Findings, Imaging Study Reporting and Imaging Study Recommendations. Radiology Cardiothoracic Imaging. Special online report. https://doi.org/10.1148/ryct.2020200214

8. Chen A, Huang J, Liao Y, Liu Z, Chen D, Yang C, Yang R, Wei X (2020) Differences in Clinical and Imaging Presentation of Pediatric Patients with COVID-19 in Comparison with Adults. Cardiothoracic Imaging. Advance online publication. https://doi.org/10.1148/ryct.2020200117

9. Tullie L, Ford K, Bisharat M, Watson T, Thakkar H, Mullassery D, Giuliani S, Blackburn S, Cross K, De Coppi P, Curry J (2020) Gastrointestinal features in children with COVID-19: an observation of varied presentation in eight children. The Lancet. Child \& adolescent health. Advance online publication. https://doi.org/10.1016/S2352-4642(20)30165-6

10. Hansell DM, Bankier AA, MacMahon H, McLoud TC, Müller NL, Remy J (2008) Fleischner Society: glossary of terms for thoracic imaging. Radiology 246(3):697-722.

https://doi.org/10.1148/radiol.2462070712

11. Belhadjer Z, Méot M, Bajolle F et al (2020) Acute heart failure in multisystem inflammatory syndrome in children (MIS-C) in the context of global SARS-CoV-2 pandemic. Circulation. Advance online publication. https://doi.org/10.1161/CIRCULATIONAHA.120.048360

12. Verdoni L, Mazza A, Gervasoni A, Martelli L, Ruggeri M, Ciuffreda M, Bonanomi E, D'Antiga L (2020) An outbreak of severe Kawasaki-like disease at the Italian epicentre of the SARS-CoV-2 epidemic: an observational cohort study. The Lancet 395(10239):1771-1778. https://doi.org/10.1016/S01406736(20)31103-X

13. Yokouchi Y, Oharaseki T, Harada M, Ihara F, Naoe S, Takahashi K (2013) Histopathological study of lymph node lesions in the acute phase of Kawasaki disease. Histopathology 62(3):387-396. https://doi.org/10.1111/his.12007

14. Chung CJ, Stein L (1998) Kawasaki disease: a review. Radiology208(1) 25-33. https://doi.org/10.1148/radiology.208.1.9646789

15. Colomba C, La Placa S, Saporito L, Corsello G, Ciccia F, Medaglia A, Romanin B, Serra N, Di Carlo P, Cascio A (2018) Intestinal Involvement in Kawasaki Disease. The Journal of pediatrics 202:186193. https://doi.org/10.1016/j.jpeds.2018.06.034 


\section{Table}

Page 10/16 
Table 1. Descriptive patient data including demographics, COVID-19 lab testing results and imaging studies and findings

Number of patients with imaging

(n)

Average Age (years)

9.2

Age range (years)

$1.3-20$

Sex

$\mathrm{F}(\%)$

21

(55\%)

M (\%)

17

(45\%)

COVID-19 testing

\begin{tabular}{ll} 
RT-PCR - / serology + & 22 \\
RT-PCR + / serology + & $(58 \%)$ \\
& 10 \\
RT-PCR + only & $(26 \%)$ \\
RT-PCR - & $4(11 \%)$ \\
RT-PCR - / serology indeterminate & $1(3 \%)$ \\
\hline
\end{tabular}

Imaging Studies

Chest Radiography (CXR)

CXR only

CXR Disease Distribution

\begin{tabular}{ll} 
Bilateral & 22 \\
& $(79 \%)$ \\
\hline Unilateral & $4(14 \%)$ \\
\hline Bilateral and Unilateral & $2(7 \%)$
\end{tabular}

CXR Disease Focality

\begin{tabular}{ll} 
Diffuse & 16 \\
& $(57 \%)$ \\
\hline Focal & $6(21 \%)$
\end{tabular}


Multifocal

$4(14 \%)$

CXR Characteristics

Peribronchial thickening

19

(68\%)

Ground glass opacities

15

(54\%)

Consolidation

$5(18 \%)$

Pleural effusion

$2(7 \%)$

Septal thickening

$1(4 \%)$

Cardiomegaly

$4(14 \%)$

Normal

$8(22 \%)$

Abdominal Radiography

$6(16 \%)$

lleus

2 (33\%)

Normal

$4(67 \%)$

Abdomen/Pelvic Sonogram

Ascites

$6(50 \%)$

Gallbladder thickening

$3(25 \%)$

Gallbladder sludge

$2(17 \%)$

Right lower quadrant bowel

$3(25 \%)$

thickening/inflammation

Right lower quadrant lymphadenopathy

$2(17 \%)$

Abdominal MRI

Terminal Ileal/rectosigmoid inflammation

1

Only ascites

1

Neck Sonogram

Lymphadenopathy 
Parietooccipital cortical abnormality - possible PRES

MR evidence of papilledema 1

Normal

\section{Figures}
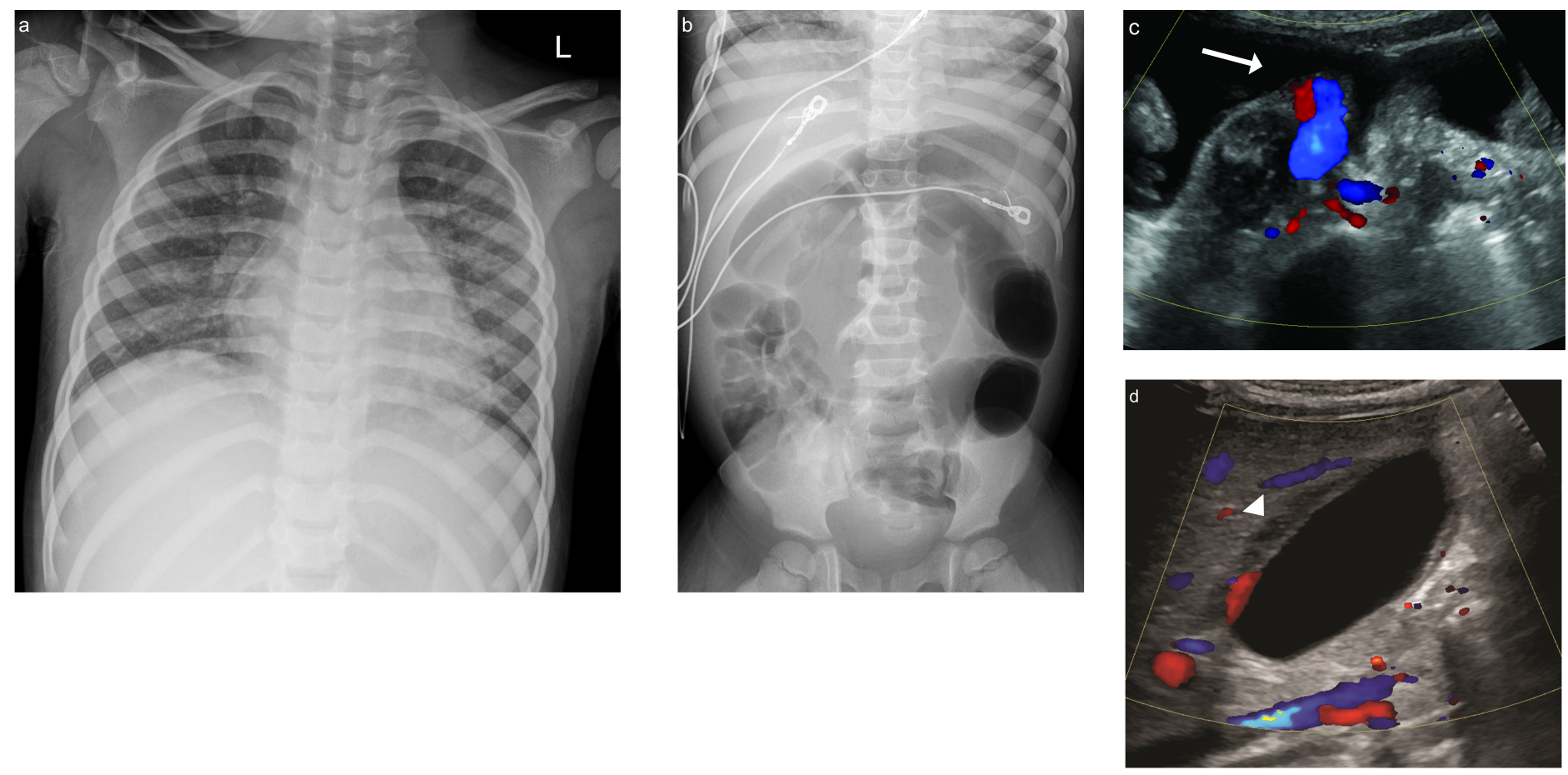

\section{Figure 1}

4 year old boy with past medical history of prematurity and asthma who presented with fever, abdominal pain, diarrhea, hypotensive shock and evidence of myocardial dysfunction with positive COVID-19 RT-PCR and serology results. a Chest radiograph demonstrates bilateral perihilar ground glass opacities and peribronchial thickening. $b$ Supine radiograph of the abdomen demonstrates abdominal distention with gaseous distention of the colon down to the rectum, suggesting colonic ileus. c Transverse sonographic image of the right lower abdominal quadrant at the level of the iliac vasculature demonstrates small volume ascites (arrow). d Sagittal sonographic image of the gallbladder demonstrates gallbladder wall thickening (arrow head) but no gallstones 

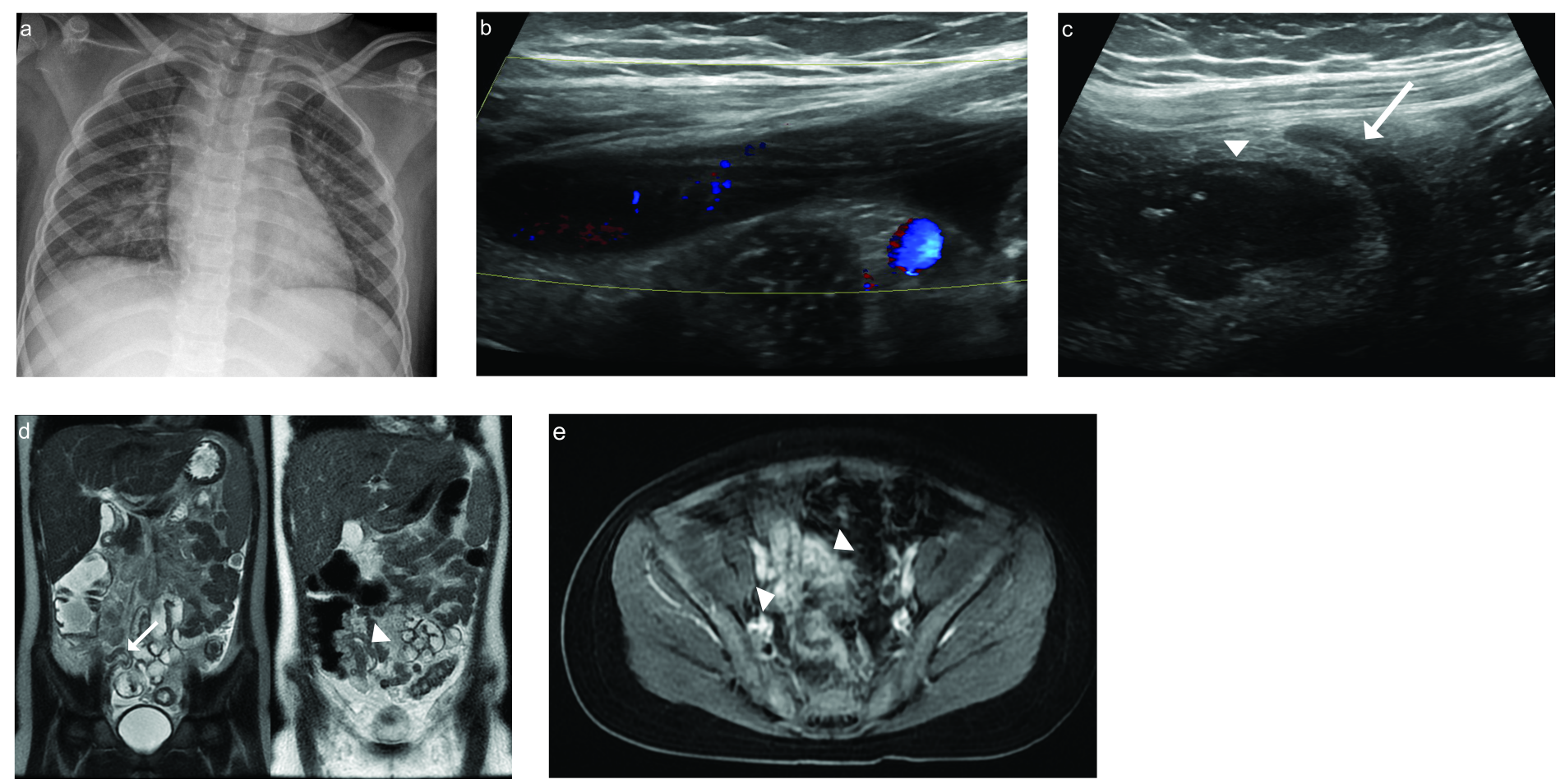

Figure 2

7 year old girl who presented with fever, abdominal pain, diarrhea, rash and evidence of myocardial dysfunction with a positive COVID-19 RT-PCR result, initially worked-up for appendicitis. a Initial chest radiograph demonstrates perihilar ground glass opacities and peribronchial thickening. b and c Transverse sonographic images of the right lower abdominal quadrant demonstrate small volume ascites and mesenteric edema in the right lower abdominal quadrant adjacent to a borderline enlarged appendix (arrow), and a thickened loop of distal ileum (arrow head). $d$ and e Abdominal MRI Coronal T2-W and axial T1-W fat saturated post gadolinium LAVA (Liver Acquisition with Volume Acceleration) images demonstrate a normal appendix (arrow) and terminal ileal and rectosigmoid wall thickening and hyperenhancement consistent with inflammation (arrow heads) 

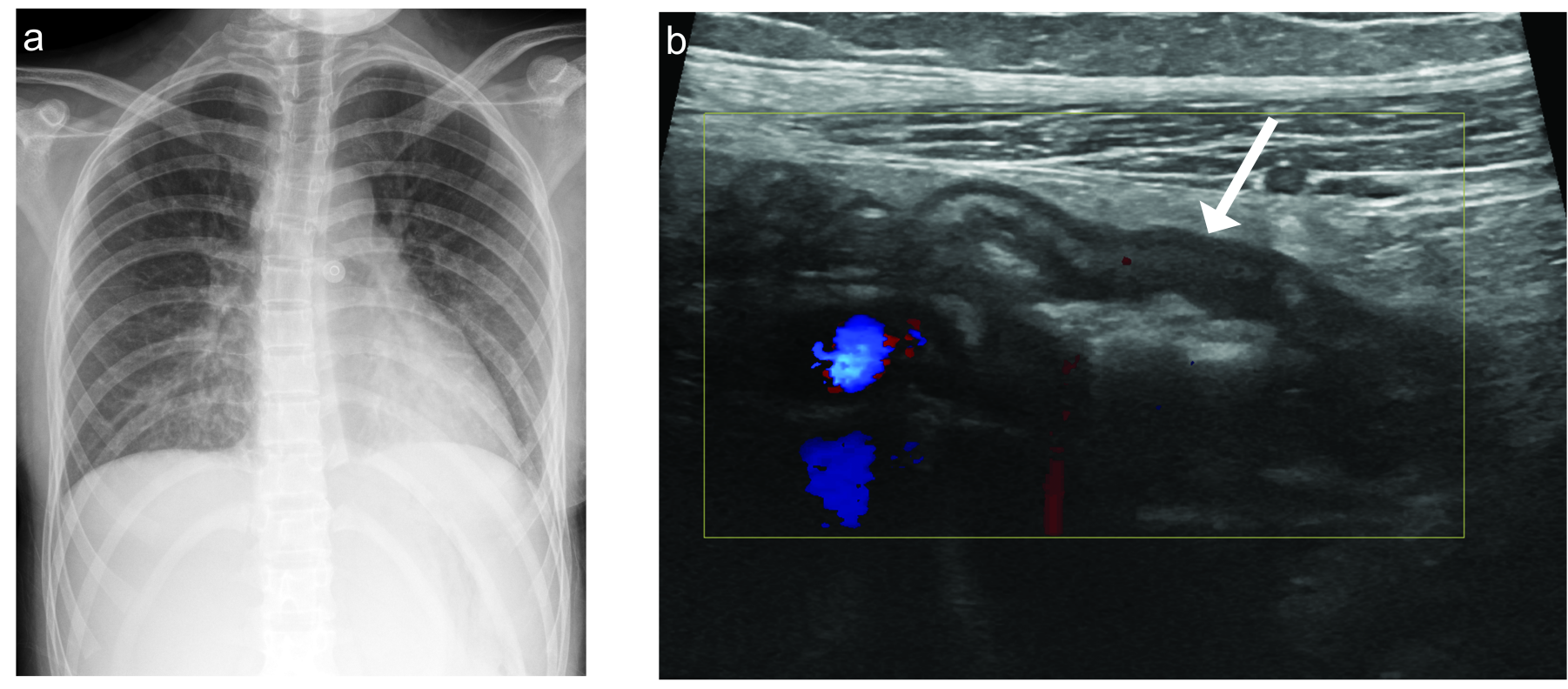

\section{Figure 3}

15 year old girl presenting with fever, abdominal pain, conjunctivitis, headaches and hypotensive shock with positive COVID-19 RT-PCR and serology results. a Chest radiograph demonstrates bilateral perihilar ground glass opacities and peribronchial thickening. b Sagittal sonographic image of the right lower abdominal quadrant demonstrates bowel wall thickening of the distal ileum (arrow) with surrounding mesenteric hyperechogenicity consistent with inflammation
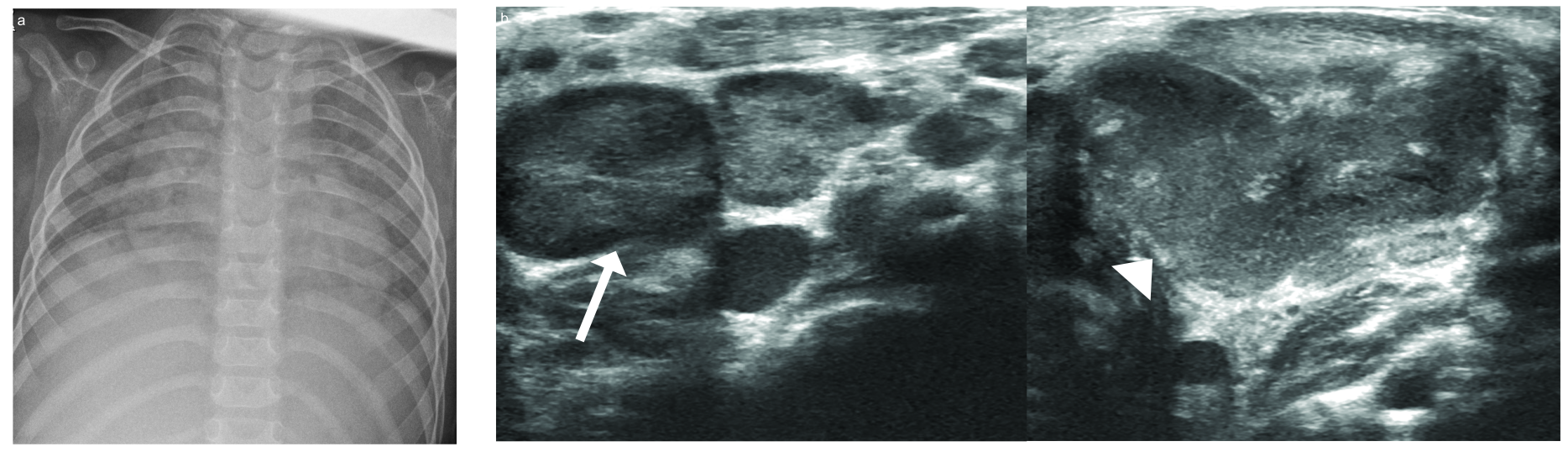

\section{Figure 4}

15 month old girl presenting with fever, cervical lymphadenopathy, rash, hand and foot swelling with skin peeling, and evidence of myocardial dysfunction with a negative COVID-19 RT-PCR result but sick family contacts that tested positive for COVID-19. a Chest radiograph demonstrates low lung volumes and diffuse airspace consolidation. b Targeted left lateral neck sonographic images demonstrate cervical 
lymphadenopathy (arrow) and an ill-defined cluster of nodes with a central avascular fluid component consistent with necrosis (arrowhead)

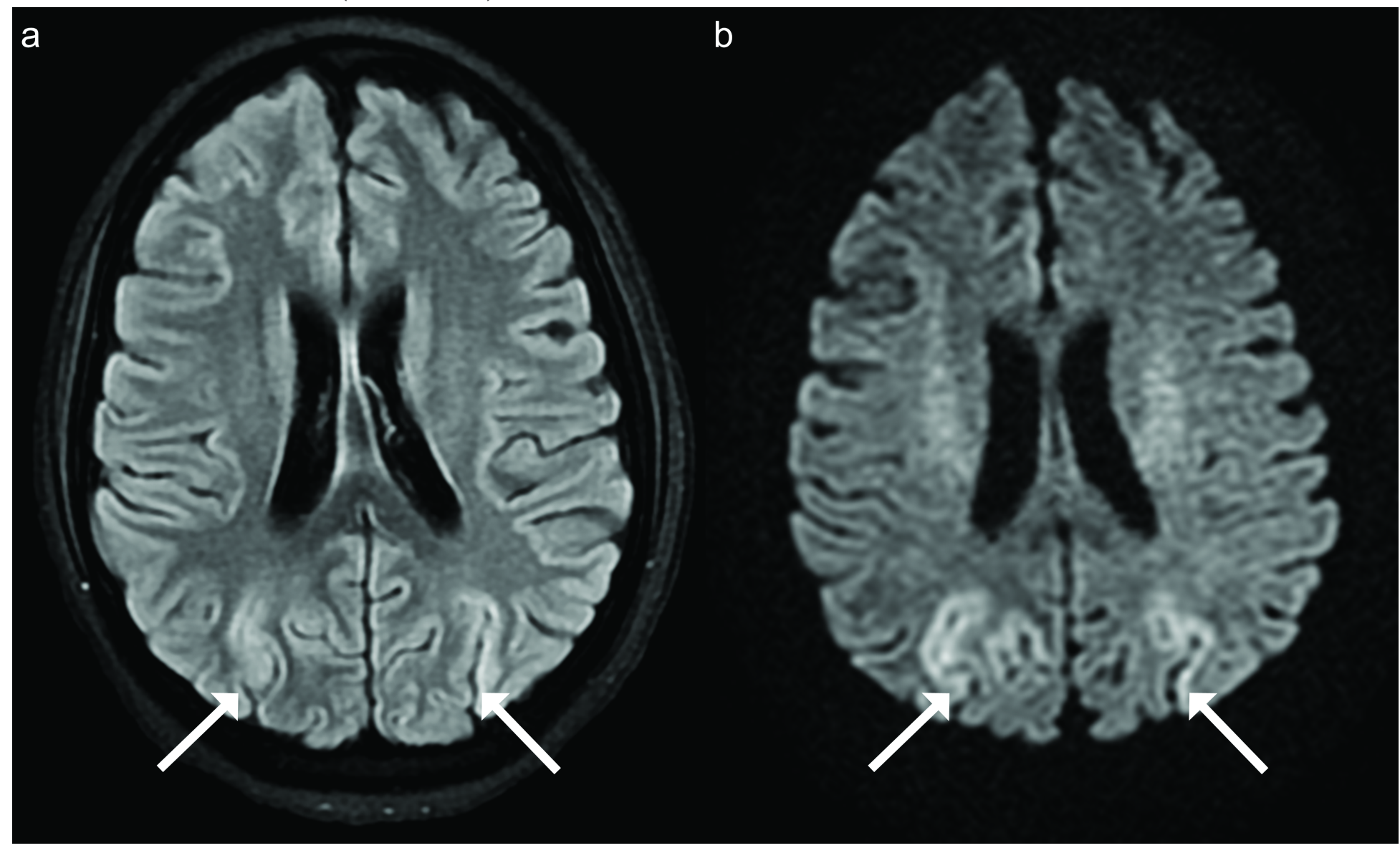

Figure 5

Fig. 514 year old girl who initially presented with typical symptoms of MIS-C including fever, abdominal pain and evidence of myocardial dysfunction with a positive COVID-19 serology result, who then developed thrombotic microangiopathy with hemolytic anemia and fluctuating mental status during admission. $a$ and b Axial FLAIR and corresponding axial DWI images through the level of the bodies of the lateral ventricles and corona radiata demonstrate FLAIR hyperintensity/restricted diffusion involving the bilateral parietooccipital cortices with mild cortical thickening 